\title{
Equivalent theorem of approximation by linear combination of weighted Baskakov-Kantorovich operators in Orlicz spaces
}

\author{
Ling-Xiong Han ${ }^{1}$, Bai-Ni Guo ${ }^{2^{*}}$ (D) and Feng Qi ${ }^{3}$
}

${ }^{*}$ Correspondence:

bai.ni.guo@gmail.com

${ }^{2}$ School of Mathematics and Informatics, Henan Polytechnic University, Jiaozuo, China

Full list of author information is

available at the end of the article

\begin{abstract}
In this paper, we introduce the Orlicz space corresponding to the Young function and, by virtue of the equivalent theorem between the modified $K$-functional and modulus of smoothness, establish the direct, inverse, and equivalent theorems for linear combination of the Jacobi weighted Baskakov-Kantorovich operators in the Orlicz spaces.
\end{abstract}

MSC: Primary 41A17; secondary 41A27; 41A35

Keywords: Equivalent theorem; Approximation; Orlicz space; Linear combination; K-functional; Weighted Jacobi-Baskakov-Kantorovich operator

\section{Motivations and main results}

In recent years, since the Orlicz spaces are more general than the classical $L_{p}$ spaces, which are composed of measurable functions $f(x)$ such that $|f(x)|^{p}$ are integrable, there is growing interest in problems of approximation in Orlicz spaces.

For proceeding smoothly, we recall from [22] some definitions and related results.

A continuous convex function $\Phi(t)$ on $[0, \infty)$ is called a Young function if

$$
\lim _{t \rightarrow 0^{+}} \frac{\Phi(t)}{t}=0 \text { and } \quad \lim _{t \rightarrow \infty} \frac{\Phi(t)}{t}=\infty .
$$

For a Young function $\Phi(t)$, its complementary Young function is denoted by $\Psi(t)$.

It is clear that the convexity of $\Phi(t)$ leads to $\Phi(\alpha t) \leq \alpha \Phi(t)$ for $\alpha \in[0,1]$. In particular, we have $\Phi(\alpha t)<\alpha \Phi(t)$ for $\alpha \in(0,1)$.

A Young function $\Phi(t)$ is said to satisfy the $\Delta_{2}$-condition, denoted by $\Phi \in \Delta_{2}$, if there exist $t_{0}>0$ and $C \geq 1$ such that $\Phi(2 t) \leq C \Phi(t)$ for $t \geq t_{0}$.

Let $\Phi(t)$ be a Young function. We define the Orlicz class $L_{\Phi}[0, \infty)$ as the collection of all Lebesgue-measurable functions $u(x)$ on $[0, \infty)$. Since the integral

$$
\rho(u, \Phi)=\int_{0}^{\infty} \Phi(|u(x)|) \mathrm{d} x
$$

(c) The Author(s) 2019. This article is distributed under the terms of the Creative Commons Attribution 4.0 International License (http://creativecommons.org/licenses/by/4.0/), which permits unrestricted use, distribution, and reproduction in any medium, provided you give appropriate credit to the original author(s) and the source, provide a link to the Creative Commons license, and indicate if changes were made. 
is finite, we define the Orlicz space $L_{\Phi}^{*}[0, \infty)$ as the linear hull of $L_{\Phi}[0, \infty)$ under the Luxembourg norm

$$
\|u\|_{(\Phi)}=\inf _{\lambda>0}\left\{\lambda: \rho\left(\frac{u}{\lambda}, \Phi\right) \leq 1\right\} .
$$

The Orlicz norm, which is equivalent to the Luxembourg norm on $L_{\Phi}^{*}[0, \infty)$, is given by

$$
\|u\|_{\Phi}=\sup _{\rho(v, \Psi) \leq 1}\left|\int_{0}^{\infty} u(x) v(x) \mathrm{d} x\right|
$$

and satisfies

$$
\|u\|_{(\Phi)} \leq\|u\|_{\Phi} \leq 2\|u\|_{(\Phi)} .
$$

Throughout this paper, we use $C$ to denote a constant independent of $n$ and $x$, which may be not necessarily the same in different cases.

Let $f \in L_{\Phi}^{*}[0, \infty)$ and $r \in \mathbb{N}$. Then the weighted $K$-functional $K_{r, \varphi}\left(f, t^{r}\right)_{w, \Phi}$, the weighted modified $K$-functional $\bar{K}_{r, \varphi}\left(f, t^{r}\right)_{w, \Phi}$, and the weighted modulus of smoothness $\omega_{r, \varphi}(f, t)_{w, \Phi}$ are given respectively by

$$
\begin{aligned}
& K_{r, \varphi}\left(f, t^{r}\right)_{w, \Phi}=\inf _{g}\left\{\|w(f-g)\|_{\Phi}+t^{r}\left\|w \varphi^{r} g^{(r)}\right\|_{\Phi}: g^{(r-1)} \in A C_{\mathrm{loc}}\right\}, \\
& \bar{K}_{r, \varphi}\left(f, t^{r}\right)_{w, \Phi}=\inf _{g}\left\{\|w(f-g)\|_{\Phi}+t^{r}\left\|w \varphi^{r} g^{(r)}\right\|_{\Phi}+t^{2 r}\left\|w g^{(r)}\right\|_{\Phi}: g^{(r-1)} \in A C_{\mathrm{loc}}\right\},
\end{aligned}
$$

and

$$
\omega_{r, \varphi}(f, t)_{w, \Phi}= \begin{cases}\sup _{0<h \leq t}\left\|w \Delta_{h \varphi}^{r}(f)\right\|_{\Phi}, & a=0, \\ \sup _{0<h \leq t}\left\|w \Delta_{h \varphi}^{r}(f)\right\|_{\Phi\left[t^{*}, \infty\right)}+\sup _{0<h \leq t^{*}}\left\|w \vec{\Delta}_{h}^{r}(f)\right\|_{\Phi\left[0,12 t^{*}\right]}, & a>0,\end{cases}
$$

where

$$
\begin{aligned}
& \Delta_{h \varphi}^{r}(f, x)=\sum_{k=0}^{r}(-1)^{k}\left(\begin{array}{l}
r \\
k
\end{array}\right) f\left(x+r\left[\frac{h \varphi(x)}{2}\right]-k h \varphi(x)\right), \\
& t^{*}=r^{2} t^{2}, \quad \varphi(x)=\sqrt{x}, \quad \varphi(x)=\sqrt{x(1+x)}, \\
& \varphi(x)=x, \quad w(x)=x^{a}(1+x)^{b}
\end{aligned}
$$

for $a, b \in \mathbb{R}$ is the Jacobi weight function, and $g^{(r-1)} \in A C_{\text {loc }}$ means that $g$ is $r-1$ times differentiable and $g^{(r-1)}$ is absolutely continuous in every closed finite interval $[c, d] \subset[0, \infty)$.

Between the weighted modulus of smoothness and the weighted modified $\bar{K}$-functional, there are the following equivalent theorems.

Theorem A ([13]) Let $f \in L_{\Phi}^{*}[0, \infty)$ and $r \in \mathbb{N}$. Then there exist constants $C$ and $t_{0}$ such that

$$
\frac{\omega_{r, \varphi}(f, t)_{w, \Phi}}{C} \leq \bar{K}_{r, \varphi}\left(f, t^{r}\right)_{w, \Phi} \leq C \omega_{r, \varphi}(f, t)_{w, \Phi}
$$

for $0<t \leq t_{0}$. 
Theorem B ([12]) Let $f \in L_{\Phi}^{*}[0, \infty)$ and $r \in \mathbb{N}$. Then there exist constants $C$ and $t_{0}$ such that

$$
\frac{\omega_{r, \varphi}(f, t)_{w, \Phi}}{C} \leq K_{r, \varphi}\left(f, t^{r}\right)_{w, \Phi} \leq C \omega_{r, \varphi}(f, t)_{w, \Phi}
$$

for $0<t \leq t_{0}$

For $f \in C([0, \infty))$, the classical Baskakov operators are defined in [3] as

$$
V_{n}(f ; x)=\sum_{k=0}^{\infty} f\left(\frac{k}{n}\right) v_{n, k}(x)
$$

where $v_{n, k}(x)=\left(\begin{array}{c}n+k-1 \\ k\end{array}\right) \frac{x^{k}}{(1+x)^{n+k}}, k, n \in \mathbb{N}$. To approximate functions in the $L_{p}$-norm, Ditzian and Totik [5] defined the Kantorovich modifications

$$
\tilde{V}_{n}(f ; x)=(n-1) \sum_{k=0}^{\infty} v_{n, k}(x) \int_{k /(n-1)}^{(k+1) /(n-1)} f(u) \mathrm{d} u
$$

and obtained the direct inequality

$$
\left\|\tilde{V}_{n} f-f\right\|_{p} \leq C\left[\omega_{2, \varphi}\left(f, \frac{1}{n^{1 / 2}}\right)_{p}+\frac{1}{n}\|f\|_{p}\right]
$$

and the week converse inequality

$$
\left\|\tilde{V}_{n} f-f\right\|_{p}=O\left(\frac{1}{n^{\alpha / 2}}\right) \Longleftrightarrow \omega_{2, \varphi}(f, h)_{p}=O\left(h^{\alpha}\right)
$$

for $\alpha<2$, where $f \in L_{p}[0, \infty), 1 \leq p<\infty$, and $\varphi(x)=\sqrt{x(1+x)}$.

There are many approximation results on operators of the Baskakov type in the space $C[0, \infty)$ or $L_{p}[0, \infty)$. See $[1-12,14-21,23,24,28,29]$ and closely related references therein. Gupta and Acu [10] discussed a uniform estimate and established a quantitative result for the modified Baskakov-Szász-Mirakyan operators. Kumar and Acar [14] introduced a modification of generalized Baskakov-Durrmeyer operators of the Stancu type and studied their approximation properties. Goyal and Agrawal [8] introduced the Bézier variant of the generalized Baskakov-Kantorovich operators, established a direct approximation theorem with the aid of the Ditzian-Totik modulus of smoothness, and studied the rate of convergence for the functions having the derivatives of bounded variation for these operators. Zhang and Zhu [29] studied preservation properties, such as monotonicity, convexity, and smoothness, as well as those under the average, of the Baskakov-Kantorovich operators. Gadjev [7] studied the approximation of functions by the Baskakov-Kantorovich operator in the space $L_{p}[0, \infty)$ and obtained the double inequality

$$
\frac{1}{C}\left\|\tilde{V}_{n} f-f\right\|_{p} \leq \tilde{K}\left(f, \frac{1}{n}\right)_{p} \leq C \frac{\ell}{n}\left(\left\|\tilde{V}_{n} f-f\right\|_{p}+\left\|\tilde{V}_{\ell} f-f\right\|_{p}\right)
$$


for $\ell \in \mathbb{N}$ with $\ell \geq C_{1} n$, where $C_{1}$ is a positive constant, and

$$
\begin{gathered}
\tilde{K}(f, t)_{p}=\inf \left\{\|f-g\|_{p}+t\|\tilde{D} g\|_{p}: f-g \in L_{p}[0, \infty),\right. \\
\left.g \in \tilde{W}_{p}[0, \infty), \tilde{D}=\frac{\mathrm{d}}{\mathrm{d} x}\left[\varphi^{2}(x) \frac{\mathrm{d}}{\mathrm{d} x}\right]\right\}
\end{gathered}
$$

is a $K$-functional.

For $n, r \in \mathbb{N}$ such that $n \geq 2 r$, the linear combinations of the Baskakov-Kantorovich operator are defined as

$$
L_{n, r}(f ; x)=\sum_{i=0}^{2 r-1} c_{i}(n, r) \tilde{V}_{n_{i}}(f ; x)
$$

where the coefficients $c_{i}(n, r)$ only dependent of $n, r$ and satisfy the following conditions:

$$
\begin{aligned}
& n \leq n_{0} \leq n_{1}<\cdots<n_{2 r-1} \leq C_{n}, \quad \sum_{i=0}^{2 r-2} c_{i}(n, r)=1, \\
& \sum_{i=0}^{2 r-2}\left|c_{i}(n, r)\right| \leq C, \\
& \sum_{i=0}^{2 r-1} c_{i}(n, r) \tilde{V}_{n_{i}}\left((t-x)^{k} ; x\right)=0, \quad k=1,2, \ldots, 2 r-1 .
\end{aligned}
$$

There are few results on the linear combinations of the Baskakov-Kantorovich operators. In [11], we obtained approximation properties for linear combinations of modified summation operators of integral type in the Orlicz space. Basing on these conclusions, we discover in this paper approximation properties for linear combinations of the BaskakovKantorovich operators.

Our main results in this paper can be stated as the following three theorems.

Theorem 1.1 (Direct theorem) Let $f \in L_{\Phi}^{*}[0, \infty), \Psi \in \Delta_{2}, \varphi(x)=\sqrt{x(1+x)}, n, a, b \in \mathbb{N}$, and $0 \leq a, b<n-1$. Then

$$
\left\|w\left(L_{n, r}(f)-f\right)\right\|_{\Phi} \leq C \omega_{2 r, \varphi}\left(f, \frac{1}{n^{1 / 2}}\right)_{w, \Phi} .
$$

Theorem 1.2 (Inverse theorem) Let $f \in L_{\Phi}^{*}[0, \infty), n \geq 2 r, \varphi(x)=\sqrt{x(1+x)}, a, b \in \mathbb{N}$, and $0 \leq a, b<n-1$. Then

$$
\omega_{2 r, \varphi}\left(f, \frac{1}{n^{r / 2}}\right)_{w, \Phi} \leq \frac{C}{n^{r}} \sum_{k=1}^{n} k^{r-1}\left\|w\left(L_{k, r}(f)-f\right)\right\|_{\Phi} .
$$


Theorem 1.3 (Equivalence theorem) Let $f \in L_{\Phi}^{*}[0, \infty), n \geq 2 r, \varphi(x)=\sqrt{x(1+x)}, \Psi \in \Delta_{2}$, $a, b \in \mathbb{N}$, and $0 \leq a, b<n-1$. Then

$$
\begin{gathered}
\left\|w\left(L_{n, r}(f)-f\right)\right\|_{\Phi}=O\left(\psi\left(\frac{1}{n^{1 / 2}}\right)\right), \quad n \rightarrow \infty \\
\Longleftrightarrow \omega_{2 r, \varphi}(f, t)_{w, \Phi}=O(\psi(t)), \quad t \rightarrow 0^{+} .
\end{gathered}
$$

These main results are stronger than the results mentioned before.

\section{Proof of the direct theorem}

To prove the direct theorem, we need several lemmas.

Lemma 2.1 The operators $\tilde{V}_{n}(f ; x)$ defined in (1.4) satisfy

$$
\tilde{V}_{n}(1 ; x)=1 \quad \text { and } \quad \tilde{V}_{n}\left((t-x)^{2 r} ; x\right) \leq \frac{C \delta_{n}^{2 r}(x)}{n^{r}}
$$

where $\delta_{n}^{2 r}(x)=\max \left\{\varphi^{2 r}(x), \frac{1}{n^{r}}\right\}, \varphi(x)=\sqrt{x(1+x)}, r \in \mathbb{N}$, and $C$ is a positive constant.

Proof This follows from simple calculation.

Lemma 2.2 ([5]) If t locates between $x$ and $u$, then

$$
\frac{(x-t)^{2 r-1}}{\varphi^{2 r}(t)} \leq \frac{|x-u|^{2 r-1}}{\varphi^{2 r-2}(x)} \frac{1}{x}\left(\frac{1}{1+x}+\frac{1}{1+u}\right) .
$$

Lemma 2.3 ([5]) For $w(x)=x^{a}(1+x)^{b}$ and $a, b \in \mathbb{R}$, we have

$$
\frac{w(x)}{w(u)} \leq 2^{|b|}\left[\left(\frac{x}{u}\right)^{a}+\left(\frac{x}{u}\right)^{b}\right]
$$

Lemma 2.4 Let $f \in L_{\Phi}^{*}[0, \infty), w(x)=x^{a}(1+x)^{b}, a, b \in \mathbb{N}$, and $0 \leq a, b<n-1$. Then

$$
\left\|w L_{n, r}(f)\right\|_{\Phi} \leq C\|w f\|_{\Phi}
$$

Proof By Lemma 2.3 we can write

$$
\begin{aligned}
\left|w(x) \tilde{V}_{n}(f ; x)\right|= & \left|\sum_{k=0}^{\infty} v_{n, k}(x) w(x)(n-1) \int_{k /(n-1)}^{(k+1) /(n-1)} f(u) \mathrm{d} u\right| \\
\leq & \sum_{k=0}^{\infty} v_{n, k}(x)(n-1) 2^{|b|+1}\left[\left(\frac{x(n-1)}{k+1}\right)^{a}\right. \\
& \left.+\left(\frac{x(n-1)}{k+1}\right)^{b}\right] \int_{k /(n-1)}^{(k+1) /(n-1)} w(u)|f(u)| \mathrm{d} u \\
\triangleq & I_{1}+I_{2} .
\end{aligned}
$$


Using (1.1) and Jensen's inequality gives

$$
\begin{aligned}
& \left\|I_{1}\right\|_{\Phi} \leq 2\left\|I_{1}\right\|_{(\Phi)} \\
& =2 \inf _{\lambda>0}\left\{\lambda: \int_{0}^{\infty} \Phi\left(\mid \sum_{k=0}^{\infty} v_{n, k}(x)(n-1) 2^{|b|+1}\left[\frac{x(n-1)}{k+1}\right]^{a}\right.\right. \\
& \left.\left.\times \int_{k /(n-1)}^{(k+1) /(n-1)} \frac{w(u) f(u)}{\lambda} \mathrm{d} u \mid\right) \mathrm{d} x \leq 1\right\} \\
& \leq 2 \inf _{\lambda>0}\left\{\lambda: \int_{0}^{\infty} \Phi\left(\sum_{k=0}^{\infty} v_{n-a, k+a}(x)(n-1) \int_{k /(n-1)}^{(k+1) /(n-1)} \frac{C w(u)|f(u)|}{\lambda} \mathrm{d} u\right) \mathrm{d} x \leq 1\right\} \\
& =2 \inf _{\lambda>0}\left\{\lambda: \int_{0}^{\infty} \Phi\left(\sum_{k=a}^{\infty} v_{n-a, k}(x)(n-1) \int_{(k-a) /(n-1)}^{(k+1-a) /(n-1)} \frac{C w(u)|f(u)|}{\lambda} \mathrm{d} u\right) \mathrm{d} x \leq 1\right\} \\
& =2 \inf _{\lambda>0}\left\{\lambda: \int_{0}^{\infty} \Phi\left(\sum_{k=a}^{\infty} v_{n-a, k}(x)(n-1) \int_{(k-a) /(n-1)}^{(k+1-a) /(n-1)} \frac{C w(u)|f(u)|}{\lambda} \mathrm{d} u\right.\right. \\
& \left.\left.+\sum_{k=0}^{a-1} v_{n-a, k}(x)(n-1) \int_{\max \left\{0, \frac{k-a}{n-1}\right\}}^{\max \left\{0, \frac{k+1-a}{n-1}\right\}} \frac{C w(u)|f(u)|}{\lambda} \mathrm{d} u\right) \mathrm{~d} x \leq 1\right\} \\
& \leq 2 \inf _{\lambda>0}\left\{\lambda: \int_{0}^{\infty} \sum_{k=0}^{\infty} v_{n-a, k}(x) \Phi\left((n-1) \int_{\max \left\{0, \frac{k-a}{n-1}\right\}}^{\max \left\{0, \frac{k+1-a}{n-1}\right\}} \frac{C w(u)|f(u)|}{\lambda} \mathrm{d} u\right) \mathrm{d} x \leq 1\right\} \\
& =2 \inf _{\lambda>0}\left\{\lambda: \int_{0}^{\infty} \sum_{k=a}^{\infty} v_{n-a, k}(x) \Phi\left((n-1) \int_{(k-a) /(n-1)}^{(k+1-a) /(n-1)} \frac{C w(u)|f(u)|}{\lambda} \mathrm{d} u\right) \mathrm{d} x \leq 1\right\} \\
& \leq 2 \inf _{\lambda>0}\left\{\lambda: \int_{0}^{\infty} \sum_{k=a}^{\infty} v_{n-a, k}(x)(n-1) \int_{(k-a) /(n-1)}^{(k+1-a) /(n-1)} \Phi\left(\frac{C w(u)|f(u)|}{\lambda}\right) \mathrm{d} u \mathrm{~d} x \leq 1\right\} \\
& \leq 2 \inf _{\lambda>0}\left\{\lambda: \sum_{k=0}^{\infty} \frac{n-1}{n-a-1} \int_{k /(n-1)}^{(k+1) /(n-1)} \Phi\left(\frac{C w(u)|f(u)|}{\lambda}\right) \mathrm{d} u \leq 1\right\} \\
& \leq 2 \inf _{\lambda>0}\left\{\lambda: \int_{0}^{\infty} \Phi\left(\frac{C w(u)|f(u)|}{\lambda}\right) \mathrm{d} u \leq 1\right\} \\
& =C\|w f\|_{(\Phi)} \leq C\|w f\|_{\Phi} .
\end{aligned}
$$

Similarly, we have

$$
\left\|I_{2}\right\|_{\Phi} \leq C\|w f\|_{\Phi}
$$

Consequently, we arrive at

$$
\left\|w(x) \tilde{V}_{n}(f ; x)\right\|_{\Phi} \leq C\|w f\|_{\Phi} .
$$


Combining (1.5), (1.6), and (2.1), it follows that

$$
\begin{aligned}
\left\|w L_{n, r}(f)\right\|_{\Phi} & =\left\|\sum_{i=0}^{2 r-1} c_{i}(n, r) w \tilde{V}_{n_{i}}(f)\right\|_{\Phi} \leq \sum_{i=0}^{2 r-1}\left\|c_{i}(n, r) w \tilde{V}_{n_{i}}(f)\right\|_{\Phi} \\
& \leq \sum_{i=0}^{2 r-1}\left|c_{i}(n, r)\right|\left\|w \tilde{V}_{n_{i}}(f)\right\|_{\Phi} \leq C \sum_{i=0}^{2 r-1}\left|c_{i}(n, r)\right|\|w f\|_{\Phi} \leq C\|w f\|_{\Phi} .
\end{aligned}
$$

The proof of Lemma 2.4 is complete.

Lemma $2.5([13])$ For $f \in L_{\Phi}^{*}[0, \infty)$ and $\Psi \in \Delta_{2}$, we have

$$
\|\theta(f)\|_{\Phi} \leq C\|f\|_{\Phi}
$$

where

$$
\theta(f ; x)=\sup _{\substack{0 \leq t<\infty \\ t \neq x}} \frac{1}{t-x} \int_{x}^{t} f(u) \mathrm{d} u
$$

is the Hardy-Littlewood function of $f(x)$.

We now in a position to prove Theorem 1.1.

Proof of Theorem 1.1 Let

$$
U=W_{\Phi}^{2 r}\left\{g: g^{(2 r-1)} \in A C_{\mathrm{loc}}, \varphi^{2 r} g^{(2 r)} \in L_{\Phi}^{*}[0, \infty)\right\}
$$

Taylor's formula with integral remainder of $g \in U$ gives

$$
g(u)=\sum_{i=0}^{2 r-1} \frac{1}{i !}(u-x)^{i} g^{(i)}(x)+R_{2 r}(g ; u, x)
$$

where

$$
R_{2 r}(g ; u, x)=\frac{1}{(2 r-1) !} \int_{x}^{u}(u-t)^{2 r-1} g^{(2 r)}(t) \mathrm{d} t, \quad x \in[0, \infty) .
$$

From (1.7) it follows that

$$
w(x)\left[L_{n, r}(g ; x)-g(x)\right]=w(x) L_{n, r}\left(R_{2 r}(g ; u, x) ; x\right)
$$

and

$$
\left\|w\left(L_{n, r}(g)-g\right)\right\|_{\Phi}=\left\|w L_{n, r}\left(R_{2 r}(g ; \cdot, x), x\right)\right\|_{\Phi} .
$$


We now estimate $\left|w(x) \tilde{V}_{n}\left(R_{2 r}(g ; u, x) ; x\right)\right|$. As $x \in\left[\frac{1}{n}, \infty\right)$, we have $\delta_{n}^{2 r}(x)=\varphi^{2 r}(x)$. Applying Lemma 2.2 leads to

$$
\begin{aligned}
& \left|w(x) \tilde{V}_{n}\left(R_{2 r}(g ; u, x) ; x\right)\right| \\
& =\mid w(x) \sum_{k=0}^{\infty} v_{n, k}(x)(n-1) \int_{k /(n-1)}^{(k+1) /(n-1)} \frac{1}{(2 r-1) !} \\
& \quad \times \int_{x}^{u}(u-t)^{2 r-1} g^{(2 r)}(t) \mathrm{d} t \mathrm{~d} u \mid \\
& \leq w(x) \sum_{k=0}^{\infty} v_{n, k}(x)(n-1) \int_{k /(n-1)}^{(k+1) /(n-1)} \frac{1}{(2 r-1) !} \frac{(u-x)^{2 r}}{\varphi^{2 r-2}(x)} \\
& \quad \times\left[\frac{1}{x(1+x)}+\frac{1}{x(1+u)}\right]\left[\frac{1}{w(u)}+\frac{1}{w(x)}\right] \mathrm{d} u\left|\theta\left(w \delta_{n}^{2 r} g^{(2 r)} ; x\right)\right| \\
& \triangleq\left(J_{1}+J_{2}+J_{3}+J_{4}\right)\left|\theta\left(w \delta_{n}^{2 r} g^{(2 r)} ; x\right)\right| .
\end{aligned}
$$

From Cauchy's integral inequality $[25,26]$ and Lemma 2.1 it follows that

$$
\begin{aligned}
J_{1}= & \sum_{k=0}^{\infty} v_{n, k}(x)(n-1) \int_{k /(n-1)}^{(k+1) /(n-1)} \frac{1}{(2 r-1) !} \frac{(u-x)^{2 r}}{\varphi^{2 r}(x)} \frac{w(x)}{w(u)} \mathrm{d} u \\
\leq & \frac{1}{(2 r-1) !} \frac{1}{\varphi^{2 r}(x)}\left[\sum_{k=0}^{\infty} v_{n, k}(x)(n-1) \int_{k /(n-1)}^{(k+1) /(n-1)} \frac{w^{2}(x)}{w^{2}(u)} \mathrm{d} u\right]^{1 / 2} \\
& \times\left[\sum_{k=0}^{\infty} v_{n, k}(x)(n-1) \int_{k /(n-1)}^{(k+1) /(n-1)}(u-x)^{4 r} \mathrm{~d} u\right]^{1 / 2} \\
\leq & \frac{1}{(2 r-1) !} \frac{1}{\varphi^{2 r}(x)} \frac{C \delta_{n}^{2 r}(x)}{n^{r}}=\frac{C}{n^{r}},
\end{aligned}
$$

where

$$
\sum_{k=0}^{\infty} v_{n, k}(x)(n-1) \int_{k /(n-1)}^{(k+1) /(n-1)} \frac{w^{2}(x)}{w^{2}(u)} \mathrm{d} u \leq C .
$$

Similarly, we can also obtain

$$
\begin{aligned}
J_{2} & =\frac{1}{(2 r-1) !} \frac{w(x)}{\varphi^{2 r}(x)} \sum_{k=0}^{\infty} v_{n, k}(x)(n-1) \int_{k /(n-1)}^{(k+1) /(n-1)} \frac{(u-x)^{2 r}}{w(x)} \mathrm{d} u \\
& =\frac{1}{(2 r-1) !} \frac{1}{\varphi^{2 r}(x)} \tilde{V}_{n}\left((u-x)^{2 r} ; x\right) \leq \frac{C}{n^{r}}
\end{aligned}
$$


Now we estimate $J_{3}$. By Cauchy's integral inequality $[25,26]$ and Lemma 2.1 we derive that

$$
\begin{aligned}
J_{3} & =\frac{w(x)}{(2 r-1) ! \varphi^{2 r-2}(x) x} \sum_{k=0}^{\infty} v_{n, k}(x)(n-1) \int_{k /(n-1)}^{(k+1) /(n-1)} \frac{(u-x)^{2 r}}{(1+u) w(u)} \mathrm{d} u \\
& =\frac{w(x)(1+x)}{(2 r-1) ! \varphi^{2 r}(x)} \sum_{k=0}^{\infty} v_{n, k}(x)(n-1) \int_{k /(n-1)}^{(k+1) /(n-1)} \frac{(u-x)^{2 r}}{(1+u) w(u)} \mathrm{d} u \\
& =\frac{w_{1}(x)}{(2 r-1) ! \varphi^{2 r}(x)} \sum_{k=0}^{\infty} v_{n, k}(x)(n-1) \int_{k /(n-1)}^{(k+1) /(n-1)} \frac{(u-x)^{2 r}}{w_{1}(u)} \mathrm{d} u \\
\leq & \frac{1}{\varphi^{2 r}(x)}\left[\sum_{k=0}^{\infty} v_{n, k}(x)(n-1) \int_{k /(n-1)}^{(k+1) /(n-1)} \frac{w_{1}^{2}(x)}{w_{1}^{2}(u)} \mathrm{d} u\right]^{1 / 2} \\
& \times\left[\sum_{k=0}^{\infty} v_{n, k}(x)(n-1) \int_{k /(n-1)}^{(k+1) /(n-1)}(u-x)^{4 r} \mathrm{~d} u\right]^{1 / 2} \\
\leq & \frac{C}{n^{r}},
\end{aligned}
$$

where $w_{1}(x)=x^{a}(1+x)^{b+1}$.

Finally, we estimate $J_{4}$. Applying Cauchy's integral inequality $[25,26]$ and Lemma 2.1 yields

$$
\begin{aligned}
J_{4} & =\frac{1}{(2 r-1) !} \frac{w(x)}{\varphi^{2 r-2}(x)} \sum_{k=0}^{\infty} v_{n, k}(x)(n-1) \int_{k /(n-1)}^{(k+1) /(n-1)} \frac{(u-x)^{2 r}}{w(x)} \frac{1}{x(1+u)} \mathrm{d} u \\
\leq & \frac{1}{x \varphi^{2 r-2}(x)}\left[\sum_{k=0}^{\infty} v_{n, k}(x)(n-1) \int_{k /(n-1)}^{(k+1) /(n-1)}(u-x)^{4 r} \mathrm{~d} u\right]^{1 / 2} \\
& \times\left[\sum_{k=0}^{\infty} v_{n, k}(x)(n-1) \int_{k /(n-1)}^{(k+1) /(n-1)} \frac{\mathrm{d} u}{(1+u)^{2}}\right]^{1 / 2} \\
\leq & \frac{1}{x \varphi^{2 r-2}(x)} \frac{C \delta_{n}^{2 r}(x)}{n^{r}} \frac{\sqrt{2}}{1+x} \leq \frac{C}{n^{r}},
\end{aligned}
$$

where

$$
\sum_{k=0}^{\infty} v_{n, k}(x)(n-1) \int_{k /(n-1)}^{(k+1) /(n-1)} \frac{\mathrm{d} u}{(1+u)^{2}} \leq \frac{2}{(1+x)^{2}}
$$

From the previous inequalities and Lemma 2.5 it follows that

$$
\begin{aligned}
\left\|w \tilde{V}_{n}\left(R_{2 r}(g ; \cdot, x) ; x\right)\right\|_{\Phi\left[\frac{1}{n}, \infty\right)} & \leq \frac{C}{n^{r}}\left\|\theta\left(w \delta_{n}^{2 r} g^{(2 r)}\right)\right\|_{\Phi\left[\frac{1}{n}, \infty\right)} \\
& \leq \frac{C}{n^{r}}\left\|w \delta_{n}^{2 r} g^{(2 r)}\right\|_{\Phi\left[\frac{1}{n}, \infty\right)}
\end{aligned}
$$


For $x \in\left[0, \frac{1}{n}\right)$, we have $\delta_{n}^{2 r}(x)=\frac{1}{n^{r}}$. Accordingly,

$$
\begin{aligned}
& \left|w(x) \tilde{V}_{n}\left(R_{2 r}(g ; u, x) ; x\right)\right| \\
& \quad \leq \sum_{k=0}^{\infty} v_{n, k}(x)(n-1) \int_{k /(n-1)}^{(k+1) /(n-1)} \frac{w(x) n^{r}}{(2 r-1) !}(u-x)^{2 r}\left[\frac{1}{w(u)}+\frac{1}{w(x)}\right] \mathrm{d} u\left|\theta\left(w \delta_{n}^{2 r} g^{(2 r)}, x\right)\right| \\
& \quad \triangleq\left(E_{1}+E_{2}\right)\left|\theta\left(w \delta_{n}^{2 r} g^{(2 r)} ; x\right)\right| .
\end{aligned}
$$

Using Cauchy's integral inequality $[25,26]$ and Lemma 2.1 yields

$$
\begin{aligned}
E_{1}= & \frac{w(x) n^{r}}{(2 r-1) !} \sum_{k=0}^{\infty} v_{n, k}(x)(n-1) \int_{k /(n-1)}^{(k+1) /(n-1)} \frac{(u-x)^{2 r}}{w(u)} \mathrm{d} u \\
\leq & \frac{n^{r}}{(2 r-1) !}\left[\sum_{k=0}^{\infty} v_{n, k}(x)(n-1) \int_{k /(n-1)}^{(k+1) /(n-1)}(u-x)^{4 r} \mathrm{~d} u\right]^{1 / 2} \\
& \times\left[\sum_{k=0}^{\infty} v_{n, k}(x)(n-1) \int_{k /(n-1)}^{(k+1) /(n-1)} \frac{w^{2}(x)}{w^{2}(u)} \mathrm{d} u\right]^{1 / 2} \\
\leq & \frac{n^{r}}{(2 r-1) !} \frac{C}{n^{r}} \delta_{n}^{2 r}(x)=\frac{C}{n^{r}}
\end{aligned}
$$

and

$$
\begin{aligned}
E_{2} & =\frac{w(x) n^{r}}{(2 r-1) !} \sum_{k=0}^{\infty} v_{n, k}(x)(n-1) \int_{k /(n-1)}^{(k+1) /(n-1)} \frac{(u-x)^{2 r}}{w(x)} \mathrm{d} u \\
& =\frac{n^{r}}{(2 r-1) !} \tilde{V}_{n}\left((u-x)^{2 r} ; x\right) \leq \frac{C}{n^{r}}
\end{aligned}
$$

Therefore we have

$$
\left|w(x) \tilde{V}_{n}\left(R_{2 r}(g ; u, x) ; x\right)\right| \leq \frac{C}{n^{r}}\left|\theta\left(w \delta_{n}^{2 r} g^{(2 r)} ; x\right)\right|
$$

and

$$
\begin{aligned}
\left\|w \tilde{V}_{n}\left(R_{2 r}(g ; \cdot, x) ; x\right)\right\|_{\Phi\left[0, \frac{1}{n}\right)} & \leq \frac{C}{n^{r}}\left\|\theta\left(w \delta_{n}^{2 r} g^{(2 r)}\right)\right\|_{\Phi\left[0, \frac{1}{n}\right)} \\
& \leq \frac{C}{n^{r}}\left\|w \delta_{n}^{2 r} g^{(2 r)}\right\|_{\Phi\left[0, \frac{1}{n}\right)} .
\end{aligned}
$$

Hence, by virtue of (2.3) and (2.4), we derive

$$
\left\|w \tilde{V}_{n}\left(R_{2 r}(g ; \cdot, x) ; x\right)\right\|_{\Phi[0, \infty)} \leq \frac{C}{n^{r}}\left\|w \delta_{n}^{2 r} g^{(2 r)}\right\|_{\Phi[0, \infty)},
$$

and, consequently,

$$
\begin{aligned}
\left\|w L_{n, r}\left(R_{2 r}(g ; \cdot, x) ; x\right)\right\|_{\Phi} & \leq \sum_{i=0}^{2 r-1}\left\|c_{i}(n, r) w \tilde{V}_{n_{i}}\left(R_{2 r}(g ; \cdot, x) ; x\right)\right\|_{\Phi} \\
& \leq \frac{C}{n^{r}} \sum_{i=0}^{2 r-1}\left|c_{i}(n, r)\right|\left\|w \delta_{n}^{2 r} g^{(2 r)}\right\|_{\Phi} \leq \frac{C}{n^{r}}\left\|w \delta_{n}^{2 r} g^{(2 r)}\right\|_{\Phi} .
\end{aligned}
$$


Combining this inequality with (1.2), (2.2), and Lemma 2.4 results in

$$
\begin{aligned}
\left\|w\left(L_{n, r}(f)-f\right)\right\|_{\Phi} & \leq\left\|w\left(L_{n, r}(f-g)-(f-g)\right)\right\|_{\Phi}+\left\|w\left(L_{n, r}(g)-g\right)\right\|_{\Phi} \\
& \leq C\|w(f-g)\|_{\Phi}+\frac{C}{n^{r}}\left\|w \delta_{n}^{2 r} g^{(2 r)}\right\|_{\Phi} \leq C \omega_{2 r, \varphi}\left(f, \frac{1}{\sqrt{n}}\right)_{w, \Phi} .
\end{aligned}
$$

The proof of the direct theorem is complete.

\section{Proofs of the inverse and equivalence theorems}

We first prepare several lemmas for proving Theorems 1.2 and 1.3.

Lemma 3.1 If $\in L_{\Phi}^{*}[0, \infty), n \geq 2 r, a, b \in \mathbb{N}$, and $0 \leq a, b<n-1$, then

$$
\left\|w \varphi^{2 r} L_{n, r}^{(2 r)}(f)\right\|_{\Phi} \leq C n^{r}\|w f\|_{\Phi}
$$

Proof From [7, Eq. (16)] it follows that

$$
\tilde{V}_{n}^{(2 r)}(f ; x)=\frac{(n+2 r-1) !}{(n-1) !} \sum_{k=0}^{\infty} \Delta^{2 r} a_{k}(n-1) v_{n+2 r, k}(x)
$$

where

$$
a_{k}(n-1)=(n-1) \int_{k /(n-1)}^{(k+1) /(n-1)} f(u) \mathrm{d} u, \quad \Delta a_{k}=a_{k+1}-a_{k}, \quad \Delta^{m} a_{k}=\Delta\left(\Delta^{m-1} a_{k}\right)
$$

By Lemma 2.3 it follows that

$$
\begin{aligned}
w(x) & \varphi^{2 r}(x) \tilde{V}_{n}^{(2 r)}(f ; x) \\
= & \frac{(n+2 r-1) !}{(n-1) !} \sum_{k=0}^{\infty} \Delta^{2 r} a_{k}(n-1) v_{n+2 r, k}(x) x^{r}(1+x)^{r} w(x) \\
\leq & C n^{r} \sum_{k=0}^{\infty}\left|\sum_{i=0}^{2 r}\left(\begin{array}{c}
2 r \\
i
\end{array}\right)(-1)^{i} a_{k+(2 r-i)}(n-1)\right| v_{n, k+r}(x) w(x) \\
\leq & C n^{r} \sum_{i=0}^{2 r}\left(\begin{array}{c}
2 r \\
i
\end{array}\right) \sum_{k=0}^{\infty} v_{n, k+r}(x) w(x)(n-1) \int_{(k+2 r-i) /(n-1)}^{(k+1+2 r-i) /(n-1)} \frac{w(u)|f(u)|}{w(u)} \mathrm{d} u \\
\leq & C n^{r} \sum_{i=0}^{2 r}\left(\begin{array}{c}
2 r \\
i
\end{array}\right) \sum_{k=0}^{\infty} v_{n, k+r}(x) 2^{|b|+1}\left[\left(\frac{(n-1) x}{k+2 r-i+1}\right)^{a}\right. \\
& \left.+\left(\frac{(n-1) x}{k+2 r-i+1}\right)^{b}\right](n-1) \int_{(k+2 r-i) /(n-1)}^{(k+1+2 r-i) /(n-1)} w(u)|f(u)| \mathrm{d} u \\
\triangleq & \sum_{i=0}^{2 r}\left(\begin{array}{c}
2 r \\
i
\end{array}\right)\left(F_{1}+F_{2}\right),
\end{aligned}
$$


where

$$
\begin{aligned}
F_{1} & =\sum_{k=0}^{\infty} v_{n, k+r}(x)\left[\frac{(n-1) x}{k+2 r-i+1}\right]^{a}(n-1) \int_{(k+2 r-i) /(n-1)}^{(k+1+2 r-i) /(n-1)} C n^{r} w(u)|f(u)| \mathrm{d} u \\
& \leq \sum_{k=0}^{\infty} v_{n-a, k+a+r}(x)(n-1) \int_{(k+2 r-i) /(n-1)}^{(k+1+2 r-i) /(n-1)} C n^{r} w(u)|f(u)| \mathrm{d} u
\end{aligned}
$$

$\left\|F_{1}\right\|_{\Phi} \leq 2\left\|F_{1}\right\|_{(\Phi)}$

$$
\begin{aligned}
& \leq 2 \inf _{\lambda>0}\left\{\lambda: \int_{0}^{\infty} \Phi\left(\sum_{k=0}^{\infty} v_{n-a, k+a+r}(x)(n-1)\right.\right. \\
& \left.\left.\times \int_{(k+2 r-i) /(n-1)}^{(k+1+2 r-i) /(n-1)} C n^{r} w(u) \frac{|f(u)|}{\lambda} \mathrm{d} u\right) \mathrm{~d} x \leq 1\right\} \\
& =2 \inf _{\lambda>0}\left\{\lambda: \int_{0}^{\infty} \Phi\left(\sum_{k=a+r}^{\infty} v_{n-a, k}(x)(n-1)\right.\right. \\
& \left.\left.\times \int_{(k+r-a-i) /(n-1)}^{(k+1+r-a-i) /(n-1)} C n^{r} w(u) \frac{|f(u)|}{\lambda} \mathrm{d} u\right) \mathrm{~d} x \leq 1\right\} \\
& \leq 2 \inf _{\lambda>0}\left\{\lambda: \int_{0}^{\infty} \Phi\left(\sum_{k=a+r}^{\infty} v_{n-a, k}(x)(n-1) \int_{(k+r-a-i) /(n-1)}^{(k+1+r-a-i) /(n-1)} C n^{r} w(u) \frac{|f(u)|}{\lambda} \mathrm{d} u\right.\right. \\
& \left.\left.+\sum_{k=0}^{a+r-1} v_{n-a, k}(x)(n-1) \int_{\max \left\{0, \frac{k+r-a-i}{n-1}\right\}}^{\max \left\{0, \frac{k+1+r-a-i}{n-1}\right\}} C n^{r} w(u) \frac{|f(u)|}{\lambda} \mathrm{d} u\right) \mathrm{~d} x \leq 1\right\} \\
& =2 \inf _{\lambda>0}\left\{\lambda: \int_{0}^{\infty} \Phi\left(\sum_{k=0}^{\infty} v_{n-a, k}(x)(n-1)\right.\right. \\
& \left.\left.\times \int_{\max \left\{0, \frac{k+r-a-i}{n-1}\right\}}^{\max \left\{0, \frac{k+1+r-a-i}{n-1}\right\}} \operatorname{Cn}^{r} w(u) \frac{|f(u)|}{\lambda} \mathrm{d} u\right) \mathrm{~d} x \leq 1\right\} \\
& \leq 2 \inf _{\lambda>0}\left\{\lambda: \int_{0}^{\infty} \sum_{k=0}^{\infty} v_{n-a, k}(x)\right. \\
& \left.\times \Phi\left((n-1) \int_{\max \left\{0, \frac{k+r-a-i}{n-1}\right\}}^{\max \left\{0, \frac{k+1+r-a-i}{n-1}\right\}} C n^{r} w(u) \frac{|f(u)|}{\lambda} \mathrm{d} u\right) \mathrm{d} x \leq 1\right\} \\
& \leq 2 \inf _{\lambda>0}\left\{\lambda: \int_{0}^{\infty} \sum_{k=a+i-r}^{\infty} v_{n-a, k}(x)(n-1)\right. \\
& \left.\times \int_{(k+r-a-i) /(n-1)}^{(k+1+r-a-i) /(n-1)} \Phi\left(C n^{r} w(u) \frac{|f(u)|}{\lambda}\right) \mathrm{d} u \mathrm{~d} x \leq 1\right\} \\
& \leq 2 \inf _{\lambda>0}\left\{\lambda: \sum_{k=a+i-r}^{\infty} \frac{n-1}{n-a-1} \int_{(k+r-a-i) /(n-1)}^{(k+1+r-a-i) /(n-1)} \Phi\left(\operatorname{Cn}^{r} w(u) \frac{|f(u)|}{\lambda}\right) \mathrm{d} u \leq 1\right\} \\
& \leq 2 \inf _{\lambda>0}\left\{\lambda: \sum_{k=0}^{\infty} \int_{k /(n-1)}^{(k+1) /(n-1)} \Phi\left(\operatorname{Cn}^{r} w(u) \frac{|f(u)|}{\lambda}\right) \mathrm{d} u \leq 1\right\} \\
& \leq C n^{r}\|w f\|_{(\Phi)} \leq C n^{r}\|w f\|_{\Phi},
\end{aligned}
$$


and, similarly,

$$
\left\|F_{2}\right\|_{\Phi} \leq C n^{r}\|w f\|_{\Phi} .
$$

Employing inequalities between (3.1) and (3.2) yields

$$
\begin{aligned}
\left\|w \varphi^{2 r}(x) \tilde{V}_{n}^{(2 r)}(f)\right\|_{\Phi} & \leq\left\|\sum_{i=0}^{2 r}\left(\begin{array}{c}
2 r \\
i
\end{array}\right)\left(F_{1}+F_{2}\right)\right\|_{\Phi} \\
& \leq\left\|\sum_{i=0}^{2 r}\left(\begin{array}{c}
2 r \\
i
\end{array}\right) F_{1}\right\|_{\Phi}+\left\|\sum_{i=0}^{2 r}\left(\begin{array}{c}
2 r \\
i
\end{array}\right) F_{2}\right\|_{\Phi} \\
& \leq \sum_{i=0}^{2 r}\left(\begin{array}{c}
2 r \\
i
\end{array}\right)\left(\left\|F_{1}\right\|_{\Phi}+\left\|F_{2}\right\|_{\Phi}\right) \leq C n^{r}\|w f\|_{\Phi} .
\end{aligned}
$$

Combining this inequality with (1.5) and (1.6) results in

$$
\left\|w \varphi^{2 r} L_{n, r}^{(2 r)}(f)\right\|_{\Phi}=\left\|\sum_{i=0}^{2 r-1} c_{i}(n, r) w \varphi^{2 r} \tilde{V}_{n_{i}}^{(2 r)}(f, x)\right\|_{\Phi} \leq C n^{r}\|w f\|_{\Phi}
$$

The proof of Lemma 3.1 is complete.

Lemma 3.2 Let $f \in L_{\Phi}^{*}[0, \infty), n \geq 2 r, a, b \in \mathbb{N}$, and $0 \leq a, b<n-1$. Then

$$
\left\|w \varphi^{2 r} L_{n, r}^{(2 r)}(f)\right\|_{\Phi} \leq C\left\|w \varphi^{2 r} f^{(2 r)}\right\|_{\Phi} .
$$

\section{Proof Since}

$$
\begin{aligned}
& \varphi^{2 r}(x) \tilde{V}_{n}^{(2 r)}(f ; x) \\
&=\frac{(n+2 r-1) !}{(n-1) !} \sum_{k=0}^{\infty} \Delta^{2 r} a_{k}(n-1) v_{n+2 r, k}(x) x^{r}(1+x)^{r} \\
&=\frac{(n+2 r-1) !}{(n-1) !} \sum_{k=0}^{\infty} \Delta^{2 r} a_{k}(n-1) v_{n+2 r, k}(x) \sum_{i=0}^{r}\left(\begin{array}{l}
r \\
i
\end{array}\right) x^{2 r-i} \\
&=\sum_{i=0}^{r}\left(\begin{array}{l}
r \\
i
\end{array}\right) \sum_{k=0}^{\infty} \Delta^{2 r} a_{k}(n-1) v_{n+i, k+2 r-i}(x) \frac{(k+2 r-i) !(n+i-1) !}{k !(n-1) !} \\
&=\sum_{i=0}^{r}\left(\begin{array}{l}
r \\
i
\end{array}\right) \sum_{k=0}^{\infty} \frac{(k+2 r-i) !(n+i-1) !}{k !(n-1) !}(n-1) \int_{0}^{1 /(n-1)} \int_{0}^{1 /(n-1)} \cdots \\
& \int_{0}^{1 /(n-1)} f^{(2 r)}\left(\frac{k}{n-1}+t_{1}+t_{2}+\cdots+t_{2 r}\right) \mathrm{d} t_{1} \mathrm{~d} t_{2} \cdots \mathrm{d} t_{2 r} v_{n+i, k+2 r-i}(x) \\
& \leq C \sum_{k=0}^{\infty}(n-1)^{2 r} \int_{0}^{1 /(n-1)} \int_{0}^{1 /(n-1)} \cdots \\
& \int_{0}^{1 /(n-1)} \sum_{i=0}^{r}\left(\begin{array}{l}
r \\
i
\end{array}\right)\left(\frac{k}{n-1}+t_{1}+t_{2}+\cdots+t_{2 r}\right)^{2 r-i}
\end{aligned}
$$




$$
\begin{aligned}
& \times\left|f^{(2 r)}\left(\frac{k}{n-1}+t_{1}+t_{2}+\cdots+t_{2 r}\right)\right| \mathrm{d} t_{1} \mathrm{~d} t_{2} \cdots \mathrm{d} t_{2 r} v_{n+i, k+2 r-i}(x) \\
\leq & C \sum_{k=0}^{\infty}(n-1)^{2 r} \int_{0}^{1 /(n-1)} \int_{0}^{1 /(n-1)} \cdots \\
& \int_{0}^{1 /(n-1)} \varphi^{2 r}\left(\frac{k}{n-1}+t_{1}+t_{2}+\cdots+t_{2 r}\right) \\
& \times\left|f^{(2 r)}\left(\frac{k}{n-1}+t_{1}+t_{2}+\cdots+t_{2 r}\right)\right| \mathrm{d} t_{1} \mathrm{~d} t_{2} \cdots \mathrm{d} t_{2 r} \sum_{i=0}^{r} v_{n+i, k+2 r-i}(x),
\end{aligned}
$$

we obtain

$$
\begin{aligned}
w(x) \varphi^{2 r}(x) \tilde{V}_{n}^{(2 r)}(f ; x) & \\
\leq & C \sum_{i=0}^{r} \sum_{k=0}^{\infty} v_{n+i, k+2 r-i}(x)(n-1)^{2 r} \int_{0}^{1 /(n-1)} \cdots \\
& \int_{0}^{1 /(n-1)} w\left(\frac{k}{n-1}+t_{1}+\cdots+t_{2 r}\right) \varphi^{2 r}\left(\frac{k}{n-1}+t_{1}+\cdots+t_{2 r}\right) \\
& \times\left|f^{(2 r)}\left(\frac{k}{n-1}+t_{1}+\cdots+t_{2 r}\right)\right| \frac{w(x)}{w\left(\frac{k}{n-1}+t_{1}+\cdots+t_{2 r}\right)} \mathrm{d} t_{1} \cdots \mathrm{d} t_{2 r} \\
\leq & C \sum_{i=0}^{r} \sum_{k=0}^{\infty} v_{n+i, k+2 r-i}(x)(n-1)^{2 r} 2^{b+1}\left[\left(\frac{n-1}{k+1}\right)^{a} x^{a}+\left(\frac{n-1}{k+1}\right)^{b} x^{b}\right] \\
& \times \int_{0}^{1 /(n-1)} \cdots \int_{0}^{1 /(n-1)} w\left(\frac{k}{n-1}+t_{1}+\cdots+t_{2 r}\right) \varphi^{2 r}\left(\frac{k}{n-1}+t_{1}+\cdots+t_{2 r}\right) \\
& \times\left|f^{(2 r)}\left(\frac{k}{n-1}+t_{1}+\cdots+t_{2 r}\right)\right| \mathrm{d} t_{1} \cdots \mathrm{d} t_{2 r} \triangleq G_{1}+G_{2},
\end{aligned}
$$

where

$$
\begin{aligned}
G_{1}= & C \sum_{i=0}^{r} \sum_{k=0}^{\infty}(n-1)^{2 r} v_{n+i, k+2 r-i}(x) 2^{b+1}\left(\frac{n-1}{k+1}\right)^{a} x^{a} \int_{0}^{1 /(n-1)} \cdots \int_{0}^{1 /(n-1)} \\
& \times w\left(\frac{k}{n-1}+t_{1}+\cdots+t_{2 r}\right) \varphi^{2 r}\left(\frac{k}{n-1}+t_{1}+\cdots+t_{2 r}\right) \\
& \times\left|f^{(2 r)}\left(\frac{k}{n-1}+t_{1}+\cdots+t_{2 r}\right)\right| \mathrm{d} t_{1} \cdots \mathrm{d} t_{2 r} \\
\leq & C \sum_{i=0}^{r} \sum_{k=0}^{\infty} v_{n+i-a, k+2 r+a-i}(x)(n-1)^{2 r} \int_{0}^{1 /(n-1)} \cdots \\
& \ldots \int_{0}^{1 /(n-1)} w\left(\frac{k}{n-1}+t_{1}+\cdots+t_{2 r}\right) \\
& \times \varphi^{2 r}\left(\frac{k}{n-1}+t_{1}+\cdots+t_{2 r}\right)\left|f^{(2 r)}\left(\frac{k}{n-1}+t_{1}+\cdots+t_{2 r}\right)\right| \mathrm{d} t_{1} \cdots \mathrm{d} t_{2 r},
\end{aligned}
$$


and, by Jensen's inequality,

$$
\begin{aligned}
& \left\|G_{1}\right\|_{\Phi} \leq 2\left\|G_{1}\right\|_{(\Phi)} \\
& \leq 2 \inf _{\lambda>0}\left\{\lambda: \int_{0}^{\infty} \Phi\left(C \sum_{i=0}^{r} \sum_{k=0}^{\infty} v_{n+i-a, k+a+2 r-i}(x)(n-1)^{2 r} \int_{0}^{1 /(n-1)} \mathrm{d} t_{1} \cdots\right.\right. \\
& \int_{0}^{1 /(n-1)} \mathrm{d} t_{2 r-1} \int_{k /(n-1)}^{(k+1) /(n-1)} w\left(t_{1}+\cdots+t_{2 r-1}+t_{2 r}\right) \\
& \left.\left.\times \varphi^{2 r}\left(t_{1}+\cdots+t_{2 r-1}+t_{2 r}\right) \frac{\left|f^{(2 r)}\left(t_{1}+\cdots+t_{2 r-1}+t_{2 r}\right)\right|}{\lambda} \mathrm{d} t_{2 r}\right) \mathrm{~d} x \leq 1\right\} \\
& \leq 2 \inf _{\lambda>0}\left\{\lambda: \int_{0}^{\infty} \Phi\left(\sum _ { i = 0 } ^ { r } \left[\sum_{k=2 r+a-i}^{\infty} v_{n+i-a, k}(x)(n-1)^{2 r} \int_{0}^{1 /(n-1)} \mathrm{d} t_{1} \cdots\right.\right.\right. \\
& \int_{0}^{1 /(n-1)} \mathrm{d} t_{2 r-1} \int_{(k-2 r-a+i) /(n-1)}^{(k-2 r-a+1+i) /(n-1)} w\left(t_{1}+\cdots+t_{2 r-1}+t_{2 r}\right) \\
& \times \varphi^{2 r}\left(t_{1}+\cdots+t_{2 r-1}+t_{2 r}\right) \frac{C\left|f^{(2 r)}\left(t_{1}+\cdots+t_{2 r-1}+t_{2 r}\right)\right|}{\lambda} \mathrm{d} t_{2 r} \\
& +\sum_{k=0}^{2 r+a-i-1} v_{n+i-a, k}(x)(n-1)^{2 r} \int_{0}^{1 /(n-1)} \mathrm{d} t_{1} \cdots \int_{0}^{1 /(n-1)} \mathrm{d} t_{2 r-1} \\
& \times \int_{\max \left\{0, \frac{k-2 r-a+i}{n-1}\right\}}^{\max \left\{0, \frac{k-2 r-a+1+i}{n-1}\right\}} w\left(t_{1}+\cdots+t_{2 r-1}+t_{2 r}\right) \varphi^{2 r}\left(t_{1}+\cdots+t_{2 r-1}+t_{2 r}\right) \\
& \left.\left.\left.\times \frac{C\left|f^{(2 r)}\left(t_{1}+\cdots+t_{2 r-1}+t_{2 r}\right)\right|}{\lambda} \mathrm{d} t_{2 r}\right]\right) \mathrm{~d} x \leq 1\right\} \\
& \leq 2 \inf _{\lambda>0}\left\{\lambda: \int_{0}^{\infty} \frac{1}{2^{r}} \sum_{i=0}^{r}\left(\begin{array}{l}
r \\
i
\end{array}\right) \sum_{k=0}^{\infty} v_{n+i-a, k}(x)(n-1)^{2 r-1} \int_{0}^{1 /(n-1)} \mathrm{d} t_{1} \ldots\right. \\
& \int_{0}^{1 /(n-1)} \mathrm{d} t_{2 r-1} \Phi\left((n-1) \int_{\max \left\{0, \frac{k-2 r-a+i}{n-1}\right\}}^{\max \left\{0, \frac{k-2 r-a+1+i}{r-1}\right\}} C 2^{r} w\left(t_{1}+\cdots+t_{2 r-1}+t_{2 r}\right)\right. \\
& \left.\left.\times \varphi^{2 r}\left(t_{1}+\cdots+t_{2 r-1}+t_{2 r}\right) \frac{\left|f^{(2 r)}\left(t_{1}+\cdots+t_{2 r-1}+t_{2 r}\right)\right|}{\lambda} \mathrm{d} t_{2 r}\right) \mathrm{~d} x \leq 1\right\} \\
& \leq 2 \inf _{\lambda>0}\left\{\lambda: \int_{0}^{\infty} \frac{1}{2^{r}} \sum_{i=0}^{r}\left(\begin{array}{l}
r \\
i
\end{array}\right) \sum_{k=0}^{\infty} v_{n+i-a, k+2 r+a-i}(x)(n-1)^{2 r} \int_{0}^{1 /(n-1)} \mathrm{d} t_{1} \cdots\right. \\
& \int_{0}^{1 /(n-1)} \mathrm{d} t_{2 r-1} \int_{k /(n-1)}^{(k+1) /(n-1)} \Phi\left(C w\left(t_{1}+\cdots+t_{2 r-1}+t_{2 r}\right)\right. \\
& \left.\left.\times \varphi^{2 r}\left(t_{1}+\cdots+t_{2 r-1}+t_{2 r}\right) \frac{\left|f^{(2 r)}\left(t_{1}+\cdots+t_{2 r-1}+t_{2 r}\right)\right|}{\lambda}\right) \mathrm{d} t_{2 r} \mathrm{~d} x \leq 1\right\} \\
& \leq 2 \inf _{\lambda>0}\left\{\lambda: \frac{1}{2^{r}} \sum_{i=0}^{r}\left(\begin{array}{l}
r \\
i
\end{array}\right) C_{1} \sum_{k=0}^{\infty}(n-1)^{2 r-1} \int_{0}^{1 /(n-1)} \mathrm{d} t_{1} \cdots \int_{0}^{1 /(n-1)} \mathrm{d} t_{2 r-1}\right. \\
& \times \int_{k /(n-1)}^{(k+1) /(n-1)} \Phi\left(C w\left(t_{1}+\cdots+t_{2 r-1}+t_{2 r}\right) \varphi^{2 r}\left(t_{1}+\cdots+t_{2 r-1}+t_{2 r}\right)\right.
\end{aligned}
$$




$$
\begin{aligned}
& \left.\left.\times \frac{\left|f^{(2 r)}\left(t_{1}+\cdots+t_{2 r-1}+t_{2 r}\right)\right|}{\lambda}\right) \mathrm{d} t_{2 r} \leq 1\right\} \\
\leq & 2 \inf _{\lambda>0}\left\{\lambda: \sum_{k=0}^{\infty}(n-1)^{2 r-1} \int_{0}^{1 /(n-1)} \mathrm{d} t_{1} \cdots \int_{0}^{1 /(n-1)} \mathrm{d} t_{2 r-1}\right. \\
& \times \int_{k /(n-1)}^{(k+1) /(n-1)} \Phi\left(w\left(t_{1}+\cdots+t_{2 r-1}+t_{2 r}\right) \varphi^{2 r}\left(t_{1}+\cdots+t_{2 r-1}+t_{2 r}\right) C\right. \\
& \left.\left.\times C_{1} \frac{\left|f^{(2 r)}\left(t_{1}+\cdots+t_{2 r-1}+t_{2 r}\right)\right|}{\lambda}\right) \mathrm{d} t_{2 r} \leq 1\right\} \\
\leq & 2 \inf _{\lambda>0}\left\{\lambda: \int_{0}^{\infty} \Phi\left(C w(t) \varphi^{2 r}(t) \frac{\left|f^{(2 r)}(t)\right|}{\lambda}\right) \mathrm{d} t \leq 1\right\} \\
\leq & C\left\|w \varphi^{2 r} f^{(2 r)}\right\|_{\Phi},
\end{aligned}
$$

where $C_{1} \geq 1$. Similarly, we have

$$
\left\|G_{2}\right\|_{\Phi} \leq C\left\|w \varphi^{2 r} f^{(2 r)}\right\|_{\Phi} .
$$

Consequently, it follows that

$$
\left\|w \varphi^{2 r} \tilde{V}_{n}^{(2 r)}(f)\right\|_{\Phi} \leq C\left\|w \varphi^{2 r} f^{(2 r)}\right\|_{\Phi}
$$

Combining this inequality with (1.5) and (1.6) yields

$$
\left\|w \varphi^{2 r} L_{n, r}^{(2 r)}(f)\right\|_{\Phi}=\left\|\sum_{i=0}^{2 r-1} c_{i}(n, r) w \varphi^{2 r} \tilde{V}_{n_{i}}^{(2 r)}(f)\right\|_{\Phi} \leq C\left\|w \varphi^{2 r} f^{(2 r)}\right\|_{\Phi} .
$$

The proof of Lemma 3.2 is complete.

We now in a position to prove Theorems 1.2 and 1.3.

Proof of Theorem 1.2 From Lemmas 3.1 and 3.2 and [27, Theorem 2.2] we obtain

$$
K_{2 r, \varphi}\left(f, \frac{1}{n^{r / 2}}\right)_{w, \Phi} \leq \frac{C}{n^{r}} \sum_{k=1}^{n} k^{r-1}\left\|w\left(L_{k, r}(f)-f\right)\right\|_{\Phi} .
$$

Application of inequality (1.3) concludes the inverse theorem.

Proof of Theorem 1.3 Using the so-called order function $\psi(t)=t^{\alpha}|\ln t|^{\beta} e^{|\ln t|^{\gamma}}$ for $0<\alpha<1$, $\beta \in \mathbb{R}$, and $\gamma<1$ and combining Theorems 1.1 and 1.2 conclude the equivalence theorem.

\section{Acknowledgements}

The authors appreciate the anonymous referees for their careful corrections to and valuable comments on the original version of this paper. 
Availability of data and materials

Not applicable.

\section{Competing interests}

The authors declare that they have no competing interests.

\section{Authors' contributions}

All authors contributed equally to the manuscript and read and approved the final manuscript.

\section{Author details}

${ }^{1}$ College of Mathematics, Inner Mongolia University for Nationalities, Tongliao, China. ${ }^{2}$ School of Mathematics and Informatics, Henan Polytechnic University, Jiaozuo, China. ${ }^{3}$ School of Mathematical Sciences, Tianjin Polytechnic University, Tianjin, China.

\section{Publisher's Note}

Springer Nature remains neutral with regard to jurisdictional claims in published maps and institutional affiliations.

Received: 15 May 2019 Accepted: 7 August 2019 Published online: 22 August 2019

\section{References}

1. Acu, A.M., Gupta, V.: Direct results for certain summation-integral type Baskakov-Szász operators. Results Math. 72(3), 1161-1180 (2017). https://doi.org/10.1007/s00025-016-0603-2

2. Agrawal, P.N., Gupta, V., Sathish Kumar, A.: Generalized Baskakov-Durrmeyer type operators. Rend. Circ. Mat. Palermo (2) 63(2), 193-209 (2014). https://doi.org/10.1007/s12215-014-0152-z

3. Baskakov, V.A.: An instance of a sequence of linear positive operators in the space of continuous functions. Dokl. Akad. Nauk SSSR (N. S.) 113, 249-251 (1957) (Russian)

4. Cao, F., Ding, C.: $L^{p}$ approximation by multivariate Baskakov-Kantorovich operators. J. Math. Anal. Appl. 348(2), 856-861 (2008). https://doi.org/10.1016/j.jmaa.2008.05.049

5. Ditzian, Z., Totik, V.: Moduli of Smoothness. Springer Series in Computational Mathematics, vol. 9. Springer, New York (1987). https://doi.org/10.1007/978-1-4612-4778-4.

6. Erençin, A.: Durrmeyer type modification of generalized Baskakov operators. Appl. Math. Comput. 218(8), 4384-4390 (2011). https://doi.org/10.1016/j.amc.2011.10.014

7. Gadjev, I.: Approximation of functions by Baskakov-Kantorovich operator. Results Math. 70(3-4), 385-400 (2016). https://doi.org/10.1007/s00025-016-0554-7

8. Goyal, M., Agrawal, P.N.: Bézier variant of the generalized Baskakov Kantorovich operators. Boll. Unione Mat. Ital. 8(4), 229-238 (2016). https://doi.org/10.1007/s40574-015-0040-2

9. Guo, S., Qi, Q., Liu, G.: The central approximation theorems for Baskakov Bézier operators. J. Approx. Theory 147(1), 112-124 (2007). https://doi.org/10.1016/j.jat.2005.02.010

10. Gupta, V., Acu, A.M.: On Baskakov-Szász-Mirakyan-type operators preserving exponential type functions. Positivity 22(3), 919-929 (2018). https://doi.org/10.1007/s11117-018-0553-x

11. Han, L.-X., Qi, F.: On approximation by linear combinations of modified summation operators of integral type in Orlicz spaces. Mathematics 7(1) (2019) Article ID 6. https://doi.org/10.3390/math7010006

12. Han, L.-X., Wu, G.: Strong converse inequality of weighted simultaneous approximation for gamma operators in Orlicz spaces $L_{\Phi}^{*}(0, \infty)$. Appl. Math. J. Chin. Univ. Ser. A 31(3), 366-378 (2016) (Chinese)

13. Han, L.-X., Wu, G.: Equivalent theorems of approximation by weighted Szász-Kantorovich-Bézier operators in Orlicz spaces. Adv. Math. (China) 47(5), 719-734 (2018)

14. Kumar, A.S., Acar, T.: Approximation by generalized Baskakov-Durrmeyer-Stancu type operators. Rend. Circ. Mat. Palermo (2) 65(3), 411-424 (2016). https://doi.org/10.1007/s12215-016-0242-1

15. Milovanović, G.V., Mursaleen, M., Nasiruzzaman, Md.: Modified Stancu type Dunkl generalization of Szász-Kantorovich operators. Rev. R. Acad. Cienc. Exactas Fís. Nat., Ser. A Mat. 112(1), 135-151 (2018). https://doi.org/10.1007/s13398-016-0369-0

16. Mursaleen, M., Al-Abied, A.A.H., Ansari, K.J.: On approximation properties of Baskakov-Schurer-Szász-Stancu operators based on q-integers. Filomat 32(4), 1359-1378 (2018). https://doi.org/10.2298/fil1804359m

17. Mursaleen, M., Ansari, K.J., Khan, A.: Approximation by Kantorovich type $q$-Bernstein-Stancu operators. Complex Anal. Oper. Theory 11(1), 85-107 (2017). https://doi.org/10.1007/s11785-016-0572-1

18. Mursaleen, M., Nasiruzzaman, Md.: Dunkl generalization of Kantorovich type Szász-Mirakjan operators via q-calculus. Asian-Eur. J. Math. 10(4), 1750077 (2017). https://doi.org/10.1142/S1793557117500772

19. Mursaleen, M., Rahman, S., Ansari, K.J.: Approximation by generalized Stancu type integral operators involving Sheffer polynomials. Carpath. J. Math. 34(2), 215-228 (2018)

20. Mursaleen, M., Rahman, S., Ansari, K.J.: On the approximation by Bézier-Păltănea operators based on Gould-Hopper polynomials. Math. Commun. 24(2), 147-164 (2019)

21. Nasiruzzaman, Md., Rao, N., Wazir, S., Kumar, R.: Approximation on parametric extension of Baskakov-Durrmeyer operators on weighted spaces. J. Inequal. Appl. 2019, 103 (2019). https://doi.org/10.1186/s13660-019-2055-1

22. Rao, M.M., Ren, Z.D.: Theory of Orlicz Space. Monographs and Textbooks in Pure and Applied Mathematics, vol. 146. Marcel Dekker, Inc., New York (1991)

23. Rao, N., Wafi, A.: Stancu-variant of generalized Baskakov operators. Filomat 31(9), 2625-2632 (2017) https://doi.org/10.2298/fil1709625r

24. Srivastava, H.M., Mursaleen, M., Nasiruzzaman, Md.: Approximation by a class of $q$-beta operators of the second kind via the Dunkl-type generalization on weighted spaces. Complex Anal. Oper. Theory 13(3), 1537-1556 (2019). https://doi.org/10.1007/s11785-019-00901-6 
25. Tian, J.-F., Ha, M.-H.: Properties of generalized sharp Hölder's inequalities. J. Math. Inequal. 11(2), 511-525 (2017). https://doi.org/10.7153/jmi-11-42

26. Tian, J.-F., Ha, M.-H., Wang, C.: Improvements of generalized Hölder's inequalities and their applications. J. Math. Inequal. 12(2), 459-471 (2018). https://doi.org/10.7153/jmi-2018-12-34

27. van Wickeren, E.: Weak-type inequalities for Kantorovitch polynomials and related operators. Nederl. Akad. Wetensch. Indag. Math. 49(1), 111-120 (1987). https://doi.org/10.1016/s1385-7258(87)80011-2

28. Verma, D.K., Gupta, V., Agrawal, P.N.: Some approximation properties of Baskakov-Durrmeyer-Stancu operators. Appl. Math. Comput. 218(11), 6549-6556 (2012). https://doi.org/10.1016/j.amc.2011.12.031

29. Zhang, C., Zhu, Z:: Preservation properties of the Baskakov-Kantorovich operators. Comput. Math. Appl. 57(9), 1450-1455 (2009). https://doi.org/10.1016/j.camwa.2009.01.027

Submit your manuscript to a SpringerOpen ${ }^{\odot}$ journal and benefit from:

- Convenient online submission

- Rigorous peer review

- Open access: articles freely available online

- High visibility within the field

- Retaining the copyright to your article

Submit your next manuscript at $\boldsymbol{s p r i n g e r o p e n . c o m ~}$ 